\title{
A Systematic Review of Integrated Traditional Chinese and Western Medicine for Managing Irritable Bowel Syndrome
}

\author{
Chun-Yan Li, ${ }^{*}$ Nurul Ain Mohd Tahir ${ }^{\dagger+}$ and Shu-Chuen $\mathrm{Li}^{*}$ \\ *Department of Gastroenterology \\ The First Affiliated Hospital of Dalian Medical University \\ Dalian 116011, P. R. China \\ ${ }^{\dagger}$ Faculty of Pharmacy, Universiti Kebangsaan Malaysia \\ Jalan Raja Muda Abdul Aziz \\ 50300 Kuala Lumpur, Malaysia \\ Discipline of Pharmacy and Experimental Pharmacology \\ School of Biomedical Sciences and Pharmacy \\ University of Newcastle, Callaghan, NSW 2308, Australia
}

Published 28 April 2015

\begin{abstract}
Traditional Chinese medicine (TCM) has been commonly used by Chinese practitioners to treat irritable bowel syndrome (IBS). However, the effectiveness of combining TCM with Western medicine in managing IBS has not been evaluated systematically. In this study, we evaluated the clinical effectiveness of combining TCM and Western medicine in the treatment of IBS via meta-analyses. We reviewed 72 eligible randomized controlled trials from January 2009 to December 2013 investigating the effectiveness of integrated TCM and Western medicine in the management of IBS. In the meta-analyses, the relative risks (RRs) and $95 \%$ confidence interval $(95 \% \mathrm{CI})$ were calculated using raw data from each study, and low heterogeneity was detected. When compared to the Western medicine treatment alone, our result showed TCM combined with Western interventions significantly improved IBS global symptoms (RR, 1.21; 95\%CI: 1.18-1.24). Additionally, there was no significant difference in therapeutic effects of the integrated approach in the meta-analyses involving the various IBS subtypes. Likewise, both Chinese proprietary herbal medicine plus conventional treatment and compound herbal preparations plus conventional treatment showed similar and statistically significant effects on global improvement compared with western treatment alone, with RRs of 1.22 (95\%CI: 1.14-1.30) and 1.22 (95\%CI: 1.18-1.27), respectively. These results demonstrated that treating IBS with integrated traditional Chinese and Western medicines showed better effectiveness than conventional Western medicine alone. Although
\end{abstract}

Correspondence to: Prof. Shu-Chuen Li, Discipline of Pharmacy and Experimental Pharmacology, School of Biomedical Sciences and Pharmacy, University of Newcastle, University Drive, Callaghan, NSW 2308, Australia. Tel: (+61) 02-4921-5921, Fax: (+61) 02-4921-7903, E-mail: Shuchuen.li@newcastle.edu.au 
due to the quality of the included studies, our results might possess a high risk of bias. TCM, particularly Chinese proprietary medicine, with the benefits of low-cost, easy to use, and good palatability, would be an attractive option to be used in conjunction with conventional Western medicine to manage IBS patients.

Keywords: Traditional Chinese Medicine; Integrated TCM and Western Medicine; Irritable Bowel Syndrome; Effectiveness; Systematic Review; Meta-Analysis.

\section{Introduction}

Irritable bowel syndrome (IBS) is a functional gastrointestinal disorder characterized by chronic or recurrent abdominal pain or discomfort, which is associated with disturbed defecation in the absence of morphological changes and biochemical abnormalities (Longstreth et al., 2006; Chang and Talley, 2010). Based on the Rome III diagnostic criteria (Longstreth et al., 2006), according to different bowel behaviors and IBS subtypes, they are presented as IBS-D (diarrhea-predominant), IBS-C (constipation-predominant), IBS-M (mixed), and unspecified IBS (IBS-U) (Brandt et al., 2009). It is a common disease which affects about $5-20 \%$ of the population worldwide, depending upon the diagnostic criteria applied (Liu and Hou, 2011). For example, in two of the major cities in China, the prevalence of IBS was estimated to be $7.26 \%$ (Manning criteria) or $0.82 \%$ (Rome criteria) in Beijing (Pan et al., 2000) and 11.5\% (Manning criteria) or $5.67 \%$ (Rome criteria) in Guangdong (Xiong et al., 2004). Besides suffering from the clinical symptoms and obvious negative impact on health-related quality of life, patients with this disorder would also require increased consumption of medical resources and shoulder a considerable economic burden (Quigley et al., 2006; Nellesen et al., 2013).

Despite the variety of approaches for symptom relief and quality of life improvement that have been used by clinicians and patients, symptoms of IBS are often recurrent and resistant to therapy. Many IBS sufferers, especially Chinese, who are not completely satisfied with the treatment outcomes, have turned to traditional Chinese medicine (TCM) for a remedy (Yoon et al., 2011; Wen et al., 2013). As a therapeutic discipline, TCM is derived and developed from the traditional Chinese physician's considerable clinical practice and experience over many centuries (Shergis et al., 2013; Liu et al., 2014b). In China, TCM is regularly used to treat IBS patients in either Western medicine hospitals or TCM hospitals. In the management of IBS, significant clinical therapeutic effects have been reported, with a number of TCM herbal mixtures specifically formulated based on the patients' symptoms (Wang et al., 2008).

In China, TCM has been routinely used alongside Western or allopathic Medicine in the healthcare system (Gu et al., 2013; Zhao et al., 2014). In the new version of the Chinese National Essential Medicines List issued by the Ministry of Health in 2013, there are a total of 520 kinds of medicines including 203 Chinese proprietary medicines. Compared with the last version released in 2009 (that included 102 varieties of Chinese medicine out of a total of 307 drugs), 101 new Chinese patent medicines were added as essential medicines. 
The proportion of increase of Chinese medicine in the new National Essential Medicines List really signals the intention of the Chinese Ministry of Health of putting more emphasis on using both Chinese and Western medicine to meet the demands for essential medicines.

Referring back to the management of IBS, studies have shown that TCM practitioners have accumulated rich experience and used different herbal products or concoctions in treating the main symptoms of IBS (Tang, 2009). In fact, the combination of TCM with Western medicine has been used for a long time in the treatment of IBS by Chinese practitioners (Zhang et al., 2013), however, their effectiveness has not been adequately evaluated. With the increasing constraint in health care resources and demands for accountability in health resource usage, the effectiveness of using TCM in the management of IBS needs to be systematically reviewed. The information obtained would allow for assessment of the value of extra cost involved in adding TCM to the management of IBS. In our present study, treatment of IBS by TCM combined with Western medicine has been evaluated systematically. This would provide the basis for further investigation, as well as making evidence-based recommendations regarding integrated TCM and Western medicine treatment. The objective of our study was to evaluate the clinical effectiveness of the combination of TCM and Western medicine in the treatment of IBS by systematically combining the results from retrieved studies via a meta-analysis, and to recommend a future role for the use of TCM in IBS management.

\section{Methods}

\section{Literature Search Strategy}

We searched PubMed, the Chinese publications in China National Knowledge Infrastructure (CNKI) and the Wanfang Database for articles from January 2009 to December 2013. The search terms used were "IBS" and "integrated TCM and Western medicine", or "IBS" and "combination of traditional Chinese and Western treatment". Furthermore, we also conducted a manual search for additional publications from the reference sections of the retrieved articles. No language restriction was applied.

\section{Study Selection Criteria}

Randomized controlled trials that investigated the effectiveness of integrated TCM and Western medicine (treatment group) compared with Western medicine (control group) in the management of IBS were included. The drugs in the control group could be Western medicines, while TCM plus the same Western medicine was used in the treatment group. All the medicines had to be administered orally.

Exclusion criteria were: (a) The drugs used in the control group were TCM or placebo; (b) different Western medicines were used in the treatment group and the control group; (c) drugs were not orally administered; (d) conference papers or master thesis not officially published; and (e) the same data was published in different journals. For the last category, only the latest publication would be considered. 


\section{Data Extraction and Analysis}

Data were collected independently by the reviewers. Any concerns about inclusion or exclusion criteria of a study were resolved by discussion among the reviewers. Data extracted from the studies included effectiveness, year of publication, clinical type of IBS, diagnostic criteria, types of Chinese medicines and Western medicines used, duration of treatment, and follow-up.

The relative risks (RRs) and 95\% confidence interval (95\% CI) were calculated using raw data derived from each study. Statistical analyses were carried out using Review Manager Software 5.2 (RevMan 5.2), which was the Cochrane Collaboration's program for preparing and maintaining Cochrane reviews. Meta-analysis was performed with random effects model or fixed effect model according to the level of heterogeneity when the treatment group was compared with the control group.

\section{Assessment of Trial Quality}

The quality of each study was assessed by RevMan 5.2. Publication bias analysis was evaluated via funnel plot.

\section{Results}

\section{Results of Literature Search}

A total of 108 potentially eligible publications were identified by the electronic search with 4 extra articles identified from manual search of the reference lists of retrieved articles. After culling, 72 articles involving 6,395 patients with IBS met the selection criteria and were included in this analysis. Figure 1 shows a flow diagram of the literature search and study selection process. All the included studies were carried out in China and published in Chinese.

\section{Study Characteristics}

Characteristics of the 72 included studies are shown in Table 1. The majority of the trials used Rome criteria for the diagnosis of IBS, while three trials used Chinese National criteria. Among the 72 included studies, 29 trials enrolled patients with IBS-D, 8 trials enrolled those with IBS-C, 1 trial enrolled patients with IBS-M, and the remaining 34 studies did not specify the type of IBS of the participants.

Of the 72 studies, 8 Chinese proprietary medicines were used in 12 studies, compound herbal preparations were prescribed in the remaining 60 studies with herbal formulae or modified formulae based on syndrome differentiation according to practitioners' clinical experience. These Chinese proprietary medicines included different forms (oral liquid, capsule, powder, or granules), while compound herbal preparations were of decoction formulation. Among the 12 studies which used Chinese proprietary herbal medicines, 


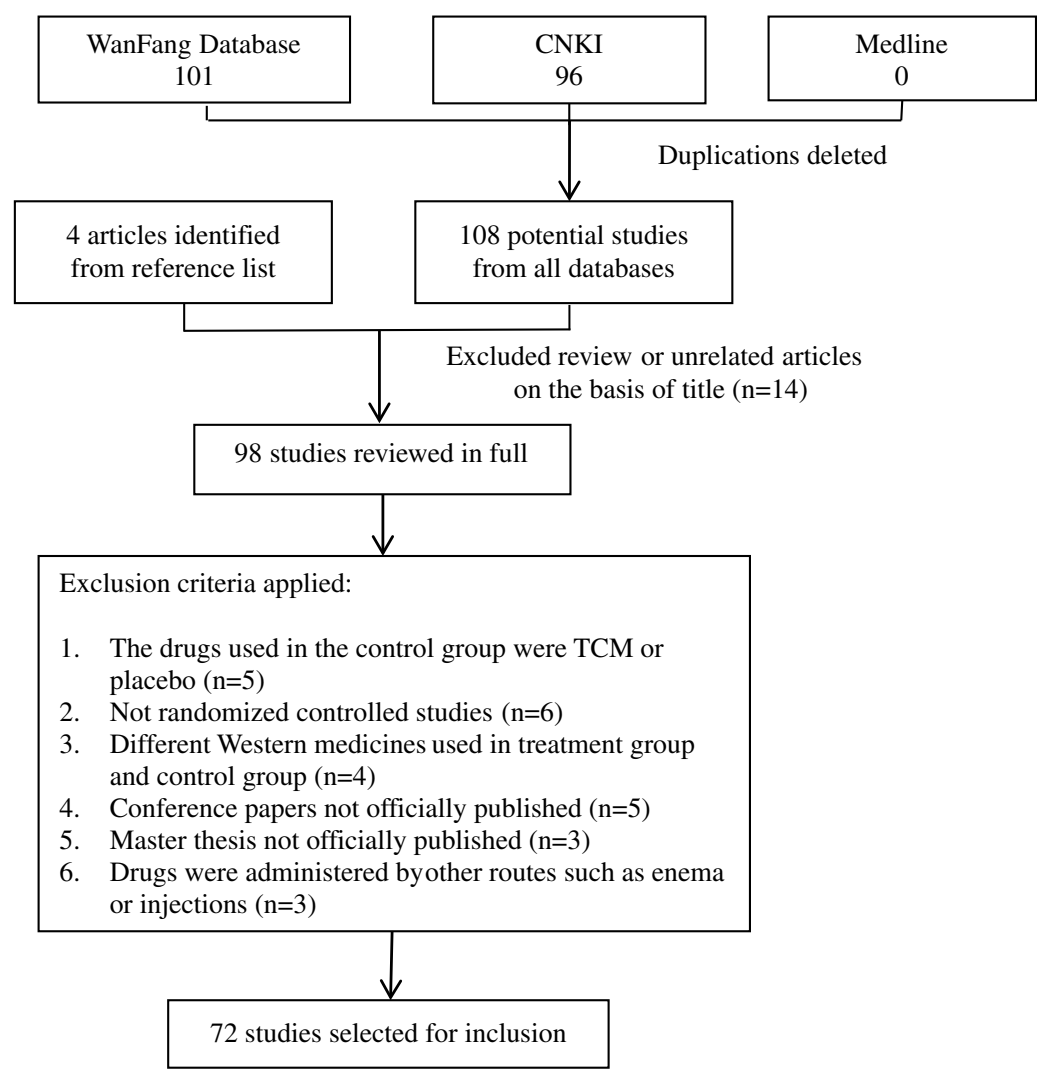

Figure 1. Flow diagram of the study selection process.

5 trials tested Gushen Changan combined with Western medicines for the treatment of IBSD, while the other 7 used different Chinese proprietary medicines for various IBS subtypes. The control interventions of Western medicines were antispasmodics, antidiarrheals, antidepressants, prokinetics, bulking agents, and probiotics; or a combination of these drugs according to the patient's symptoms.

The duration of treatment was 4 weeks in 48 studies, 1 week in 1 study, 2 weeks in 5 studies, 2-4 weeks in 2 studies, 5-8 weeks in 14 studies, while the longest period of treatment was 12 weeks in 2 studies.

\section{Risk of Bias Assessment}

Among the 72 randomized controlled studies, 4 studies used a random number table to generate the allocation sequence (Wu, 2009; Chen, 2012; Li, 2012b; Xiong, 2013), and three grouped according to the patients' treatment order (Fan, 2009; Li, 2010, 2012a), while the remaining 65 studies did not describe the sequence generation method in detail. None of the studies reported blindness or an adequate concealed allocation method of randomization. Only one study reported the withdrawal information (Li, 2012b). Using the 


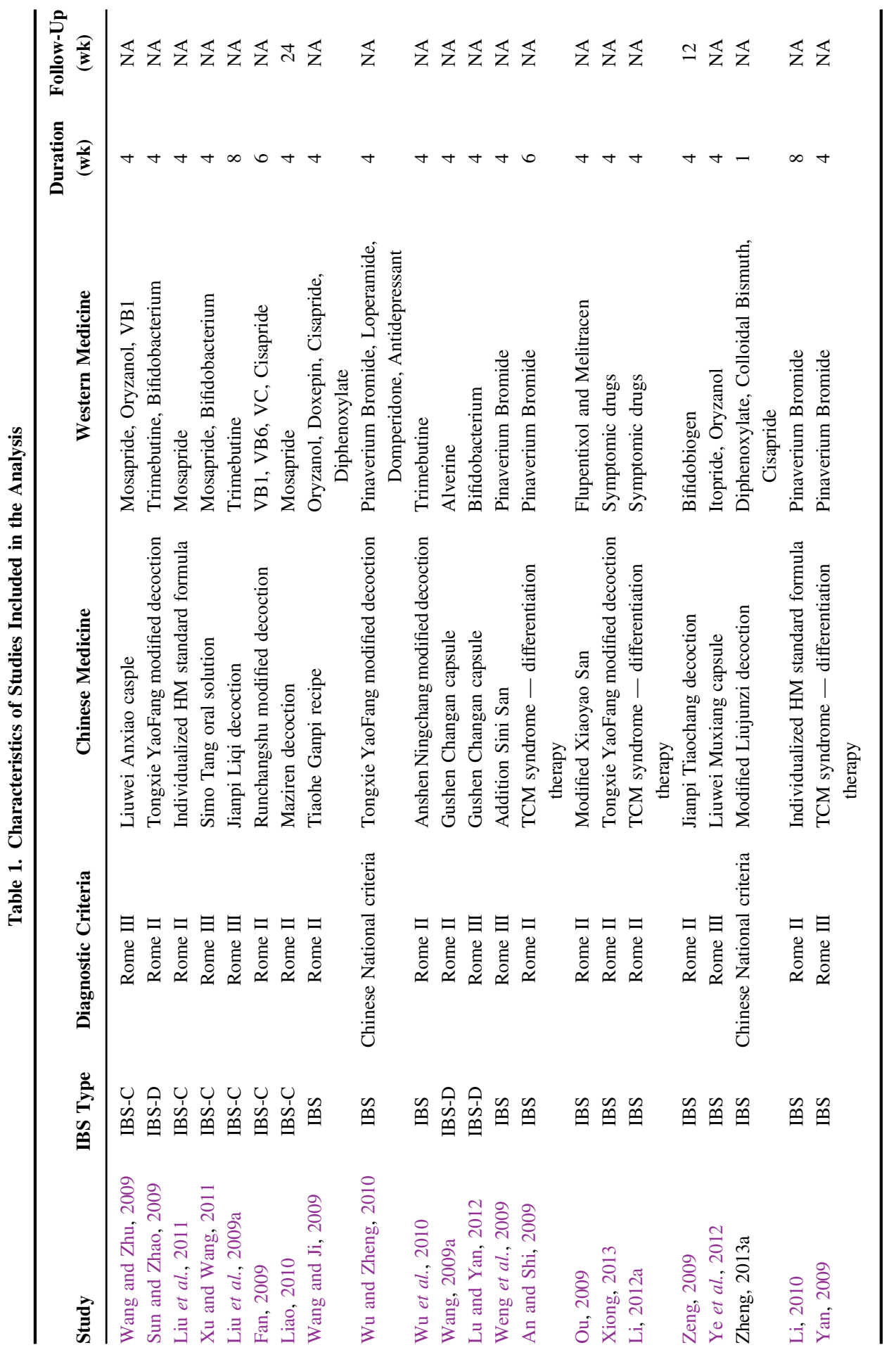


TREATMENT OF IBS WITH INTEGRATIVE THERAPIES

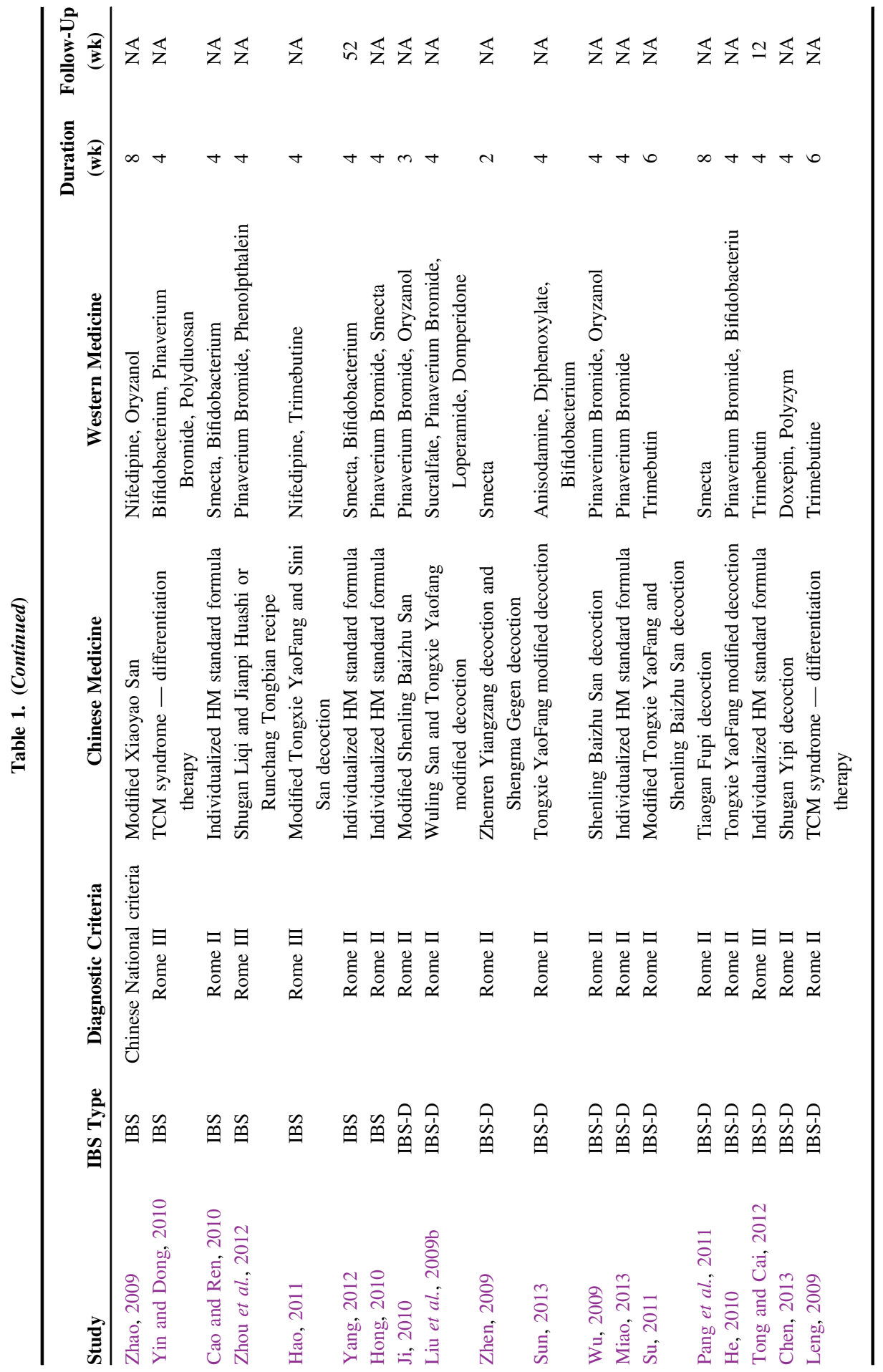




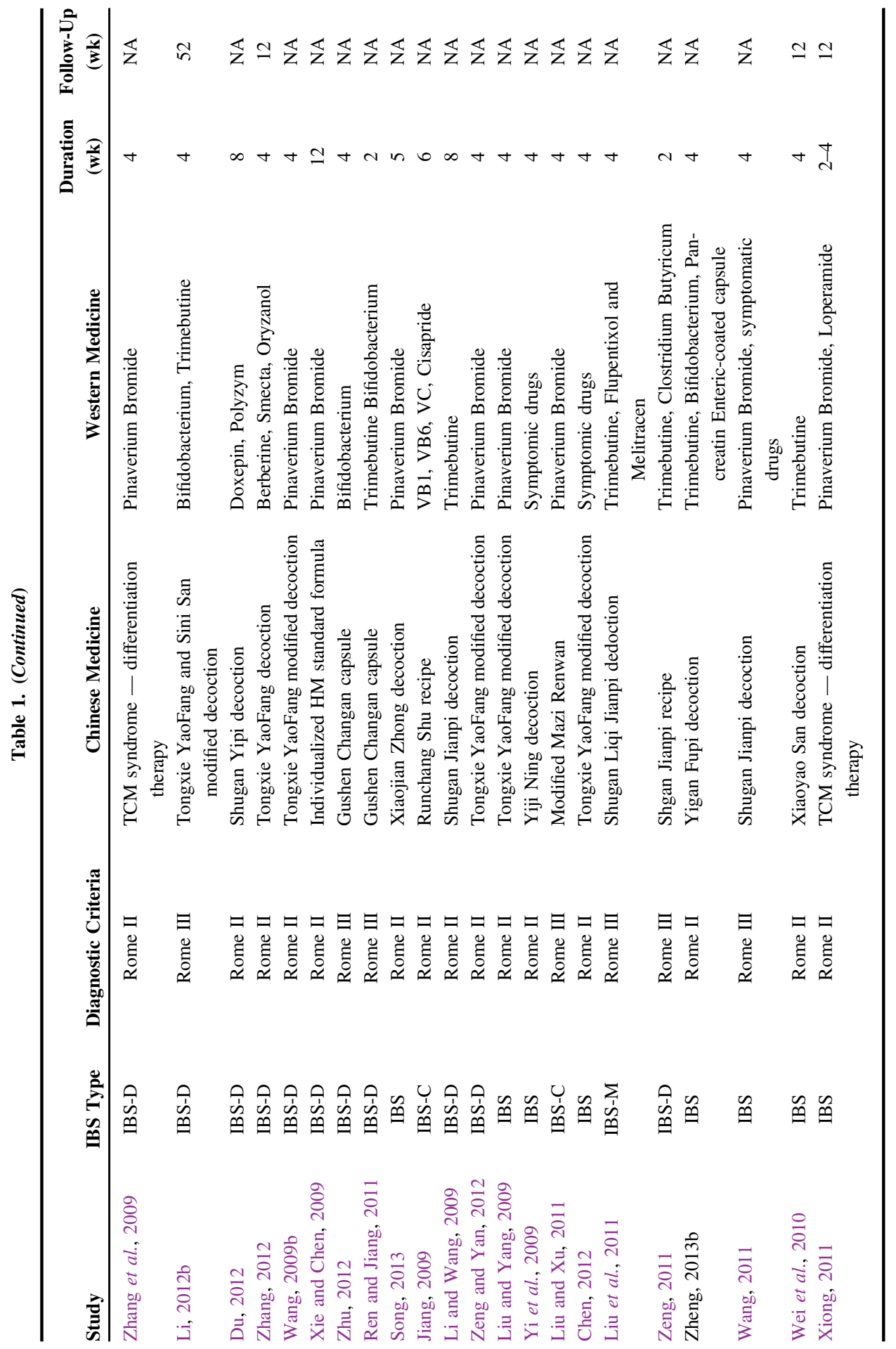




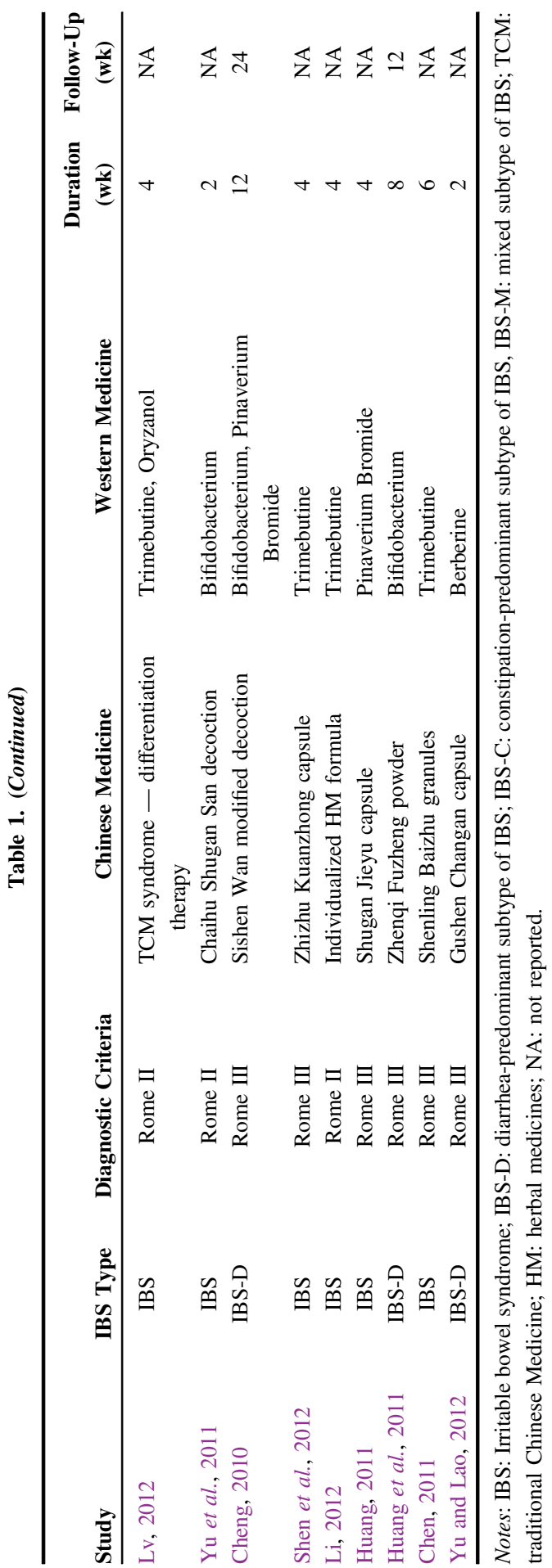


general methodological quality assessment from the Cochrane Handbook, these studies might possess high risk of bias.

\section{Effectiveness of Combination of TCM and Western Medicine}

\section{Global Symptoms of IBS}

All studies reported outcomes of the global improvement of symptoms, and the total effective rate was calculated with effective patients divided by the total number of cases (i.e., using the Intention-to-Treat analysis). Evaluation criteria was based on "Clinical Research Guidelines for New Chinese Herbal Drug" (Zheng, 2002), "Irritable bowel syndrome diagnosis and treatment of Integrative Medicine program (draft)" (Chen et al., 2005), or "Diagnostic basis of clinical disease and criteria for recovery and improvement" (Sun, 2002).

Among the 72 studies, 71 reported that the patients in the treatment group showed a statistically significant improvement in symptoms compared with control group after treatment. By contrast, only one study demonstrated an insignificant improvement in symptoms. The RRs for each study and all studies combined for the combination of TCM and Western medicine vs. Western medicine alone are shown in Fig. 2. In the randomeffects meta-analysis of all included studies, low heterogeneity $\left(\tau^{2}=0.00 ; \chi^{2}=76.44\right.$, $p=0.31 ; I^{2}=7 \%$ ) was detected. When compared to the Western medicine treatment, our meta-analysis showed TCM combined with Western medicine interventions significantly improved global symptoms of IBS (RR, 1.21; 95\%CI: 1.18-1.24).

\section{Subtype Analysis - Effectiveness in Various IBS Subtypes}

We examined the effectiveness of integrated TCM and Western medicine by different IBS subtypes. As shown in Fig. 3, the results of meta-analysis showed that compared to Western medicine treatment alone, the integrated approach was more effective in the treatment of IBS-D in the 29 trials that enrolled patients with IBS-D (RR, 1.19; 95\%CI: $1.15-1.24)$. Again, low level of heterogeneity $\left(\tau^{2}=0.00 ; \chi^{2}=33.01, p=0.24\right.$; $I^{2}=15 \%$ ) was detected. Similarly, 8 IBS-C trials also demonstrated that integrated TCM and Western medicine was therapeutically more effective than Western medicine alone (RR, 1.23; 95\%CI: 1.13-1.33) (Fig. 4). Only one study treated IBS-M and reported that the effective rate of treatment group was $95.0 \%$, which control group was $77.5 \%$ (Liu et al., 2011). There was significant difference between two groups $(p<0.05)$.

The remaining 34 studies did not specify the type of IBS in the participants and showed that the total effective rate was higher in the treatment groups compared to control groups (RR, 1.22; 95\%CI: 1.18-1.27) (Fig. 5). The effects were similar to the global improvement of symptoms of all the studies.

\section{Subgroup Analysis - Chinese Proprietary Medicines vs. Chinese Herbal Decoctions}

For the subgroup analysis, we examined the possible different efficacies between Chinese proprietary herbal medicines and compound herbal preparations. We found that both 


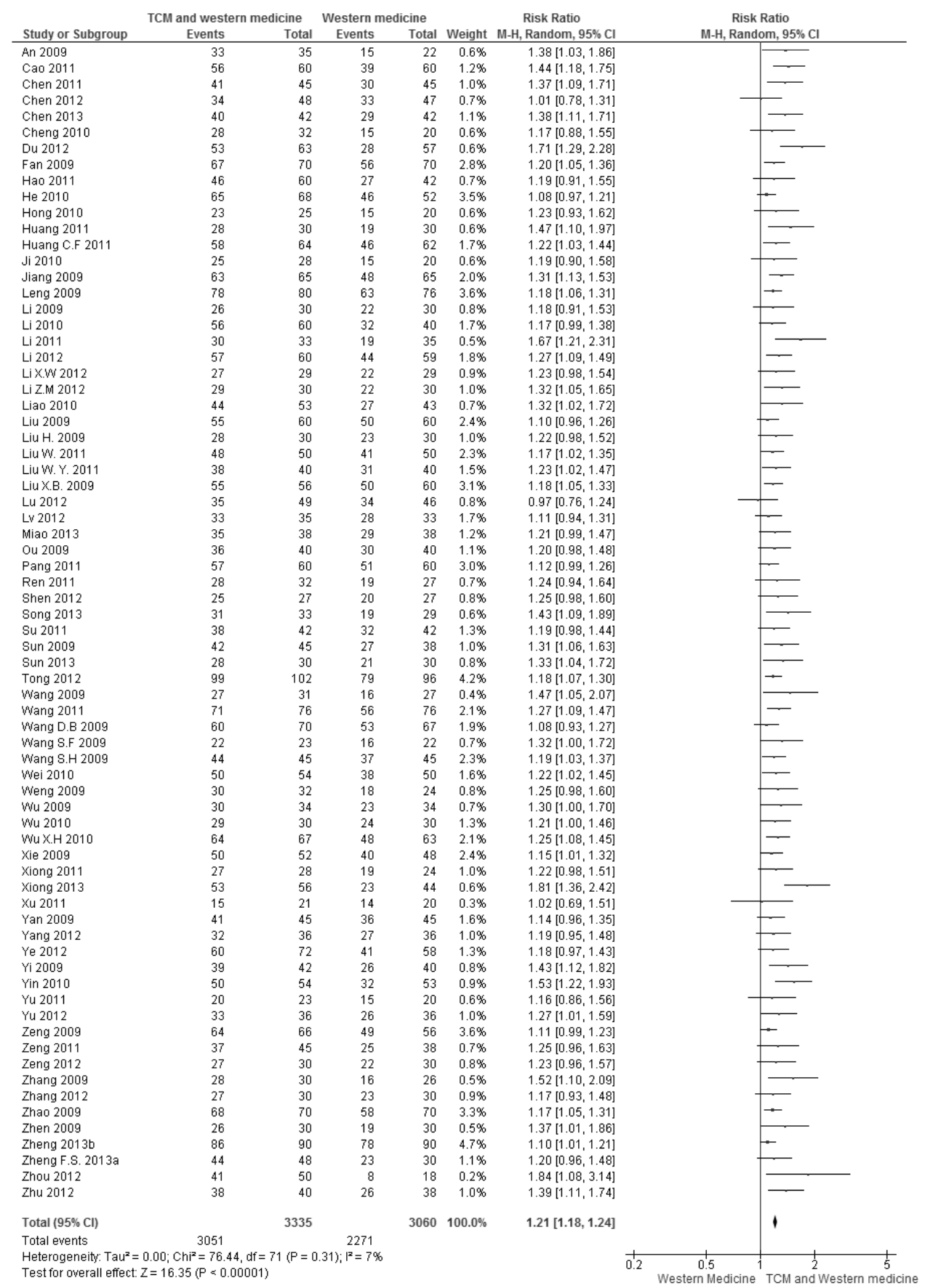

Figure 2. Comparison of TCM plus Western medicine and Western medicine for all studies. 


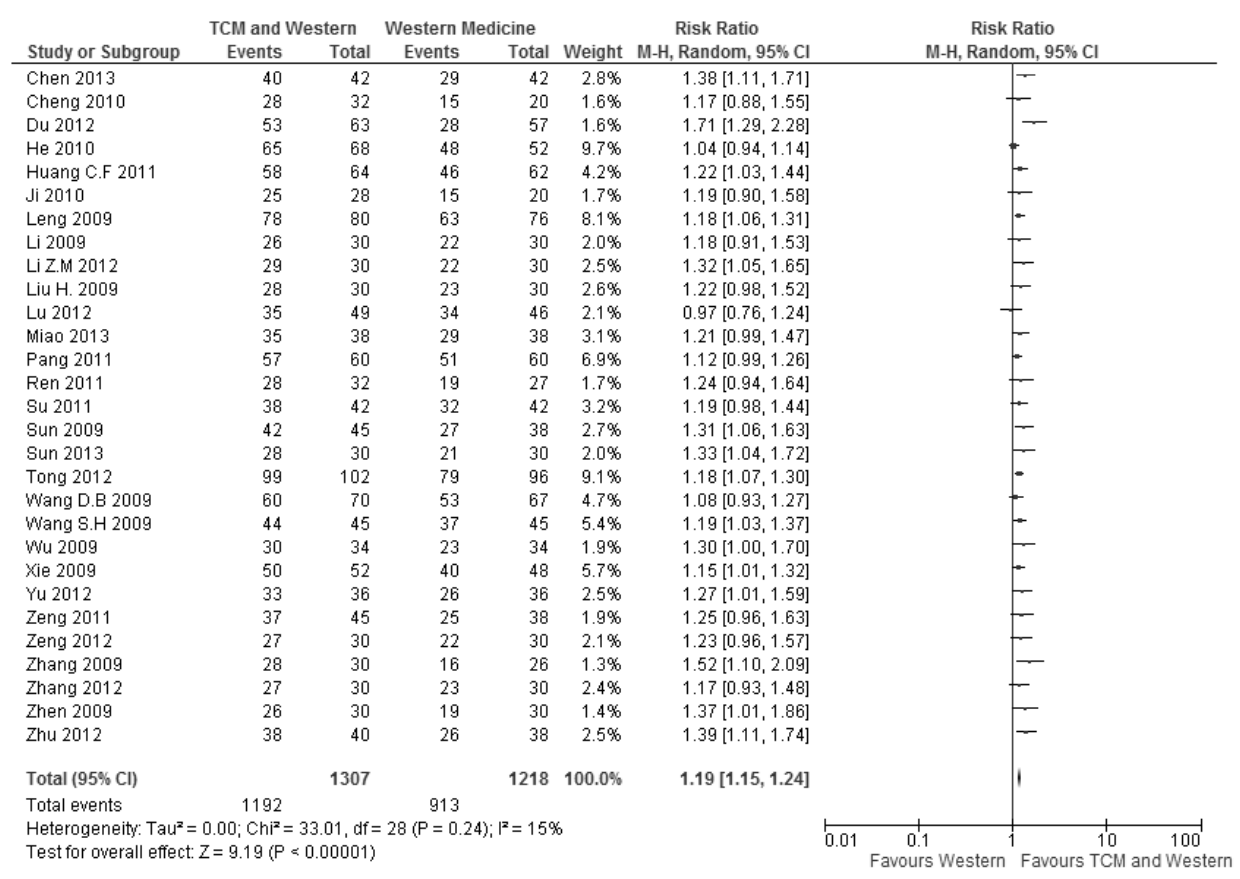

Figure 3. Comparison of TCM plus Western medicine and Western medicine in treatment of IBS-D patients.

Chinese proprietary herbal medicine plus Western medicine treatment and compound herbal preparations plus Western medicine treatment showed similar and statistically significant effects on global improvement compared with Western medicine treatment alone. RRs were 1.22 (95\%CI: $1.14-1.30)$ and 1.22 (95\%CI: 1.18-1.27), respectively. There was little heterogeneity $\left(\tau^{2}=0.00 ; \chi^{2}=11.74, p=0.38 ; I^{2}=6 \%\right)$ in Chinese proprietary herbal medicine plus conventional treatment trials, and medium heterogeneity $\left(\tau^{2}=0.01\right.$; $\chi^{2}=121.78, p<0.00001 ; I^{2}=52 \%$ ) in compound herbal preparations plus conventional treatment trials. Meta-analysis results are shown in Figs. 6 and 7. However, the number of included articles was small; therefore, further studies and analysis should be performed in future to confirm this observation.

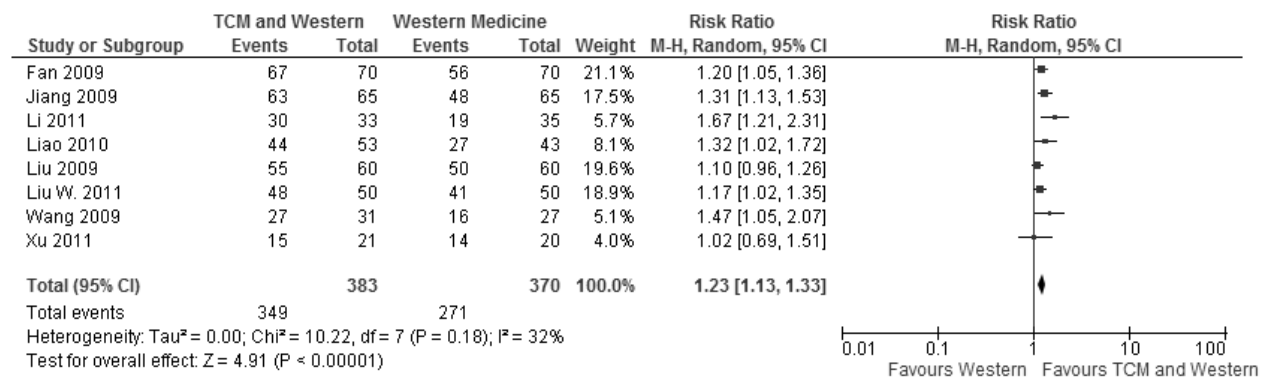

Figure 4. Comparison of TCM plus Western medicine and Western medicine in treatment of IBS-C patients. 


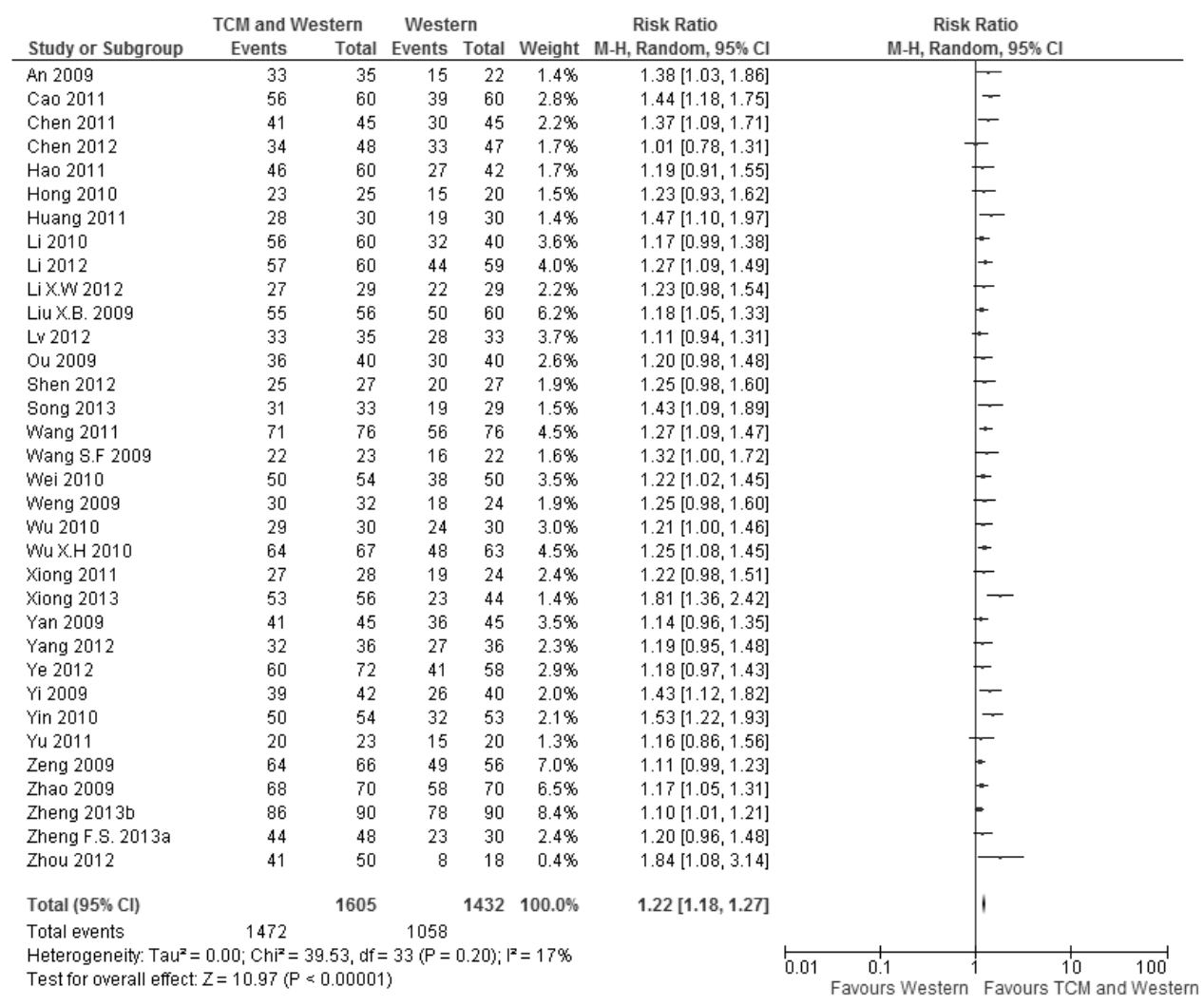

Figure 5. Comparison of TCM plus Western medicine and Western medicine for studies not specify the type of IBS.

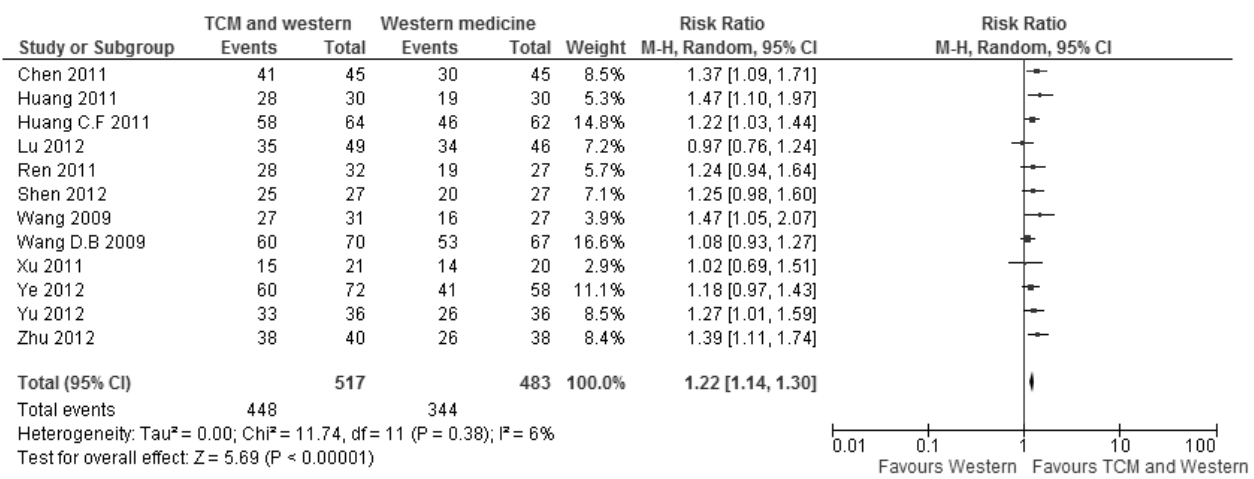

Figure 6. Comparison of Chinese proprietary herbal medicine plus Western medicine and Western medicine. 


\begin{tabular}{|c|c|c|c|c|c|c|c|c|c|}
\hline \multirow[b]{2}{*}{ Study or Subgroup } & \multicolumn{2}{|c|}{ TCM and Western } & \multicolumn{2}{|c|}{ Western Medicine } & \multirow[b]{2}{*}{ Weight } & \multirow{2}{*}{$\begin{array}{c}\text { Risk Ratio } \\
\text { M-H, Random, } 95 \% \mathrm{Cl}\end{array}$} & \multirow{2}{*}{\multicolumn{2}{|c|}{$\begin{array}{c}\text { Risk Ratio } \\
\text { M-H, Random, } 95 \% \mathrm{Cl}\end{array}$}} & \\
\hline & Events & Total & Events & Total & & & & & \\
\hline An 2009 & 33 & 35 & 15 & 22 & $1.0 \%$ & $1.38[1.03,1.86]$ & & F- & \\
\hline Cao 2011 & 56 & 60 & 39 & 60 & $1.7 \%$ & $1.44[1.18,1.75]$ & & - & \\
\hline Chen 2012 & 34 & 48 & 33 & 47 & $1.2 \%$ & $1.01[0.78,1.31]$ & & $t$ & \\
\hline Chen 2013 & 40 & 42 & 29 & 42 & $1.5 \%$ & $1.38[1.11,1.71]$ & & - & \\
\hline Cheng 2010 & 28 & 32 & 15 & 20 & $1.0 \%$ & $1.17[0.88,1.55]$ & & - & \\
\hline Du 2012 & 53 & 63 & 28 & 57 & $1.0 \%$ & $1.71[1.29,2.28]$ & & - & \\
\hline Fan 2009 & 67 & 70 & 56 & 70 & $2.5 \%$ & $1.20[1.05,1.36]$ & & - & \\
\hline Hao 2011 & 46 & 60 & 27 & 42 & $1.2 \%$ & $1.19[0.91,1.55]$ & & - & \\
\hline He 2010 & 65 & 68 & 46 & 52 & $2.7 \%$ & $1.08[0.97,1.21]$ & & - & \\
\hline Hong 2010 & 23 & 25 & 15 & 20 & $1.1 \%$ & $1.23[0.93,1.62]$ & & $\leftarrow$ & \\
\hline Ji 2010 & 25 & 28 & 15 & 20 & $1.0 \%$ & $1.19[0.90,1.58]$ & & - & \\
\hline Jiang 2009 & 63 & 65 & 48 & 65 & $2.2 \%$ & $1.31[1.13,1.53]$ & & - & \\
\hline Leng 2009 & 78 & 80 & 63 & 76 & $2.7 \%$ & $1.18[1.06,1.31]$ & & - & \\
\hline Li 2009 & 26 & 30 & 22 & 30 & $1.2 \%$ & $1.18[0.91,1.53]$ & & - & \\
\hline Li 2010 & 56 & 60 & 32 & 40 & $2.0 \%$ & $1.17[0.99,1.38]$ & & - & \\
\hline Li 2011 & 30 & 33 & 19 & 35 & $0.9 \%$ & $1.67[1.21,2.31]$ & & - & \\
\hline Li 2012 & 57 & 60 & 44 & 59 & $2.1 \%$ & $1.27[1.09,1.49]$ & & - & \\
\hline LiXW 2012 & 27 & 29 & 22 & 29 & $1.4 \%$ & $1.23[0.98,1.54]$ & & - & \\
\hline Li Z.M 2012 & 29 & 30 & 22 & 30 & $1.4 \%$ & $1.32[1.05,1.65]$ & & - & \\
\hline Liao 2010 & 44 & 53 & 27 & 43 & $1.2 \%$ & $1.32[1.02,1.72]$ & & - & \\
\hline Liu 2009 & 55 & 60 & 50 & 60 & $2.4 \%$ & $1.10[0.96,1.26]$ & & - & \\
\hline Liu H. 2009 & 28 & 30 & 23 & 30 & $1.5 \%$ & $1.22[0.98,1.52]$ & & - & \\
\hline Liu W. 2011 & 48 & 50 & 41 & 50 & $2.3 \%$ & $1.17[1.02,1.35]$ & & F & \\
\hline Liu W. Y. 2011 & 38 & 40 & 31 & 40 & $1.8 \%$ & $1.23[1.02,1.47]$ & & - & \\
\hline Liu X.B. 2009 & 55 & 56 & 50 & 60 & $2.6 \%$ & $1.18[1.05,1.33]$ & & - & \\
\hline LV 2012 & 33 & 35 & 28 & 33 & $2.0 \%$ & $1.11[0.94,1.31]$ & & - & \\
\hline Miao 2013 & 35 & 38 & 29 & 38 & $1.6 \%$ & $1.21[0.99,1.47]$ & & - & \\
\hline Ou 2009 & 36 & 40 & 30 & 40 & $1.6 \%$ & $1.20[0.98,1.48]$ & & - & \\
\hline Pang 2011 & 57 & 60 & 51 & 60 & $2.6 \%$ & $1.12[0.99,1.26]$ & & - & \\
\hline Song 2013 & 31 & 33 & 19 & 29 & $1.1 \%$ & $1.43[1.09,1.89]$ & & - & \\
\hline Su 2011 & 38 & 42 & 32 & 42 & $1.7 \%$ & $1.19[0.98,1.44]$ & & - & \\
\hline Sun 2009 & 42 & 45 & 27 & 38 & $1.5 \%$ & $1.31[1.06,1.63]$ & & - & \\
\hline Sun 2013 & 28 & 30 & 21 & 30 & $1.2 \%$ & $1.33[1.04,1.72]$ & & - & \\
\hline Tong 2012 & 99 & 102 & 79 & 96 & $2.9 \%$ & $1.18[1.07,1.30]$ & & - & \\
\hline Wang 2011 & 71 & 76 & 56 & 76 & $2.2 \%$ & $1.27[1.09,1.47]$ & & - & \\
\hline Wang S.F 2009 & 22 & 23 & 16 & 22 & $1.1 \%$ & $1.32[1.00,1.72]$ & & - & \\
\hline Wang S.H 2009 & 44 & 45 & 37 & 45 & $2.3 \%$ & $1.19[1.03,1.37]$ & & - & \\
\hline Wei 2010 & 50 & 54 & 38 & 50 & $1.9 \%$ & $1.22[1.02,1.45]$ & & - & \\
\hline Weng 2009 & 30 & 32 & 18 & 24 & $1.3 \%$ & $1.25[0.98,1.60]$ & & - & \\
\hline Wu 2009 & 30 & 34 & 23 & 34 & $1.2 \%$ & $1.30[1.00,1.70]$ & & - & \\
\hline Wu 2010 & 29 & 30 & 24 & 30 & $1.7 \%$ & $1.21[1.00,1.46]$ & & - & \\
\hline WuX.H 2010 & 64 & 67 & 48 & 63 & $2.2 \%$ & $1.25[1.08,1.45]$ & & - & \\
\hline Xie 2009 & 50 & 52 & 40 & 48 & $2.3 \%$ & $1.15[1.01,1.32]$ & & - & \\
\hline Xiong 2011 & 27 & 28 & 19 & 24 & $1.5 \%$ & $1.22[0.98,1.51]$ & & - & \\
\hline Xiong 2013 & 53 & 56 & 23 & 44 & $1.0 \%$ & $1.81[1.36,2.42]$ & & - & \\
\hline Yan 2009 & 41 & 45 & 36 & 45 & $1.9 \%$ & $1.14[0.96,1.35]$ & & - & \\
\hline Yang 2012 & 32 & 36 & 27 & 36 & $1.5 \%$ & $1.19[0.95,1.48]$ & & - & \\
\hline Yi 2009 & 39 & 42 & 26 & 40 & $1.3 \%$ & $1.43[1.12,1.82]$ & & 一 & \\
\hline Yin 2010 & 50 & 54 & 32 & 53 & $1.4 \%$ & $1.53[1.22,1.93]$ & & - & \\
\hline Yu 2011 & 20 & 23 & 15 & 20 & $1.0 \%$ & $1.16[0.86,1.56]$ & & - & \\
\hline Zeng 2009 & 64 & 66 & 49 & 56 & $2.8 \%$ & $1.11[0.99,1.23]$ & & - & \\
\hline Zeng 2011 & 37 & 45 & 25 & 38 & $1.1 \%$ & $1.25[0.96,1.63]$ & & - & \\
\hline Zeng 2012 & 27 & 30 & 22 & 30 & $1.3 \%$ & $1.23[0.96,1.57]$ & & - & \\
\hline Zhang 2009 & 28 & 30 & 16 & 26 & $0.9 \%$ & $1.52[1.10,2.09]$ & & - & \\
\hline Zhang 2012 & 27 & 30 & 23 & 30 & $1.4 \%$ & $1.17[0.93,1.48]$ & & - & \\
\hline Zhao 2009 & 68 & 70 & 58 & 70 & $2.7 \%$ & $1.17[1.05,1.31]$ & & - & \\
\hline Zhen 2009 & 26 & 30 & 19 & 30 & $0.9 \%$ & $1.37[1.01,1.86]$ & & - & \\
\hline Zheng 2013b & 86 & 90 & 78 & 80 & $3.4 \%$ & $0.98[0.93,1.04]$ & & 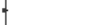 & \\
\hline Zheng F.S. 2013a & 44 & 48 & 23 & 30 & $1.5 \%$ & $1.20[0.96,1.48]$ & & - & \\
\hline Zhou 2012 & 41 & 50 & 8 & 18 & $0.4 \%$ & $1.84[1.08,3.14]$ & & & \\
\hline Total $(95 \% \mathrm{Cl})$ & & 2818 & & 2567 & $100.0 \%$ & $1.22[1.18,1.27]$ & & 1 & \\
\hline Total events & 2603 & & 1927 & & & & & & \\
\hline $\begin{array}{l}\text { Heterogeneity: Tau }{ }^{2} \\
\text { Test for overall effec }\end{array}$ & $\begin{array}{l}0.01 ; \mathrm{Chi}^{2}= \\
\mathrm{Z}=11.54(\mathrm{~F}\end{array}$ & $\begin{array}{l}21.78, d \\
0.0000\end{array}$ & $f=59(P<0$ & 101); $1^{2}$ & $=52 \%$ & & $\begin{array}{|cc|}0.01 & 0.1 \\
& \text { Favours Western }\end{array}$ & Favours TC & $\begin{array}{c}100 \\
\text { nd Wester }\end{array}$ \\
\hline
\end{tabular}

Figure 7. Comparison of compound herbal preparations plus Western medicine and Western medicine.

\section{Follow-Up and Adverse Events}

The additional outcome measures were number and type of adverse events reported in six studies (Fan, 2009; Jiang, 2009; Wang, 2009a; Ren and Jiang, 2011; Chen, 2012; Xiong, 2013). These included allergic reaction, headache, nausea, diarrhea, fatigue, and loss of 


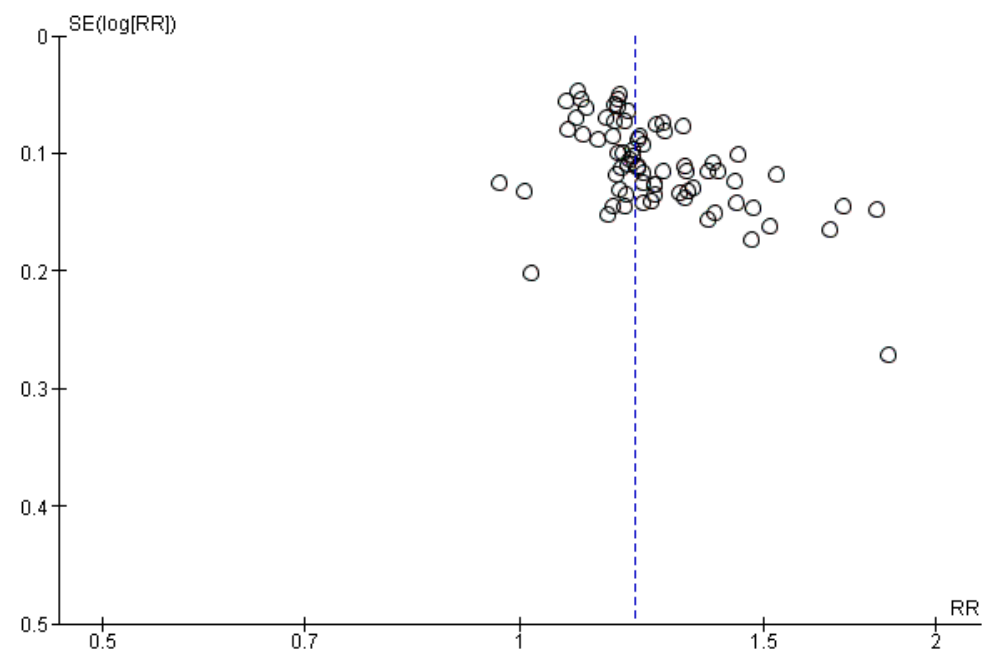

Figure 8. Funnel plot of comparison of TCM plus Western medicine and Western medicine for all studies.

appetite, which occurred both in the treatment and control groups in similar proportion. No serious adverse events were reported from the integrative therapies or Western medicines, with only tolerable minor side effects which disappeared without special treatment. Another 11 studies reported that no side effects occurred during the trial period.

Ten trials reported follow-up assessment ranging from three months to one year after the treatment. Of these studies, seven reported lower recurrence rates in the treatment group compared to the control group (Zeng, 2009; Zhao, 2009; Cheng, 2010; Wei et al., 2010; Li, 2012b; Yang, 2012; Zhang, 2012). No trials reported any cost-effectiveness information.

\section{Publication Bias Analysis}

The funnel plot indicated an asymmetry (Fig. 8), which could either be interpreted as there being no studies which showed the integrated approach in IBS management to be inferior to Western medicine treatment alone, or studies with negative results were not published.

\section{Discussion}

Although IBS is a common disease, there is still no universally accepted satisfactory treatment. Patients with IBS often have absenteeism, reduced health-related quality of life, more frequent physician visits, and greater medication use. All these negative effects of IBS naturally impose great social and economic burden on the patients and society (Quigley et al., 2006; Ringel et al., 2009; Nellesen et al., 2013). Hence, more effective treatments for IBS are needed to relieve symptoms, improve health-related quality of life, and reduce health care utilization.

Due to the wide range of the symptoms that may be experienced, the available conventional pharmacological approaches are mainly targeted at symptomatic relief, such as 
treatments with antispasmodics for abdominal pain, antidiarrheals for diarrhea, antidepressants for depression and anxiety, and prokinetics and bulking agents for constipation (Longstreth et al., 2006; Brandt et al., 2009). However, the use of these multiple medications has been limited by side effects, expensive drug costs, and high recurrence rate. The low levels of satisfaction with the treatment of these conventional medicines, in recent years, have caused an increasing number of patients and practitioners turning to alternative medicine for symptom reduction, particularly Chinese herbal medicines or integrated Chinese and Western medicine for remedy (Rahimi and Abdollahi, 2012; Grundmann and Yoon, 2014; Liu et al., 2014a).

In this respect, clinical trials have shown that TCM therapies or TCM combined with Western medicine might offer better symptom improvement to some patients with IBS. Among these trials, a number of Chinese proprietary medicines were used more frequently according to syndrome subtypes (Li et al., 2013). Although a previous review of herbal medicine alone showed a beneficial effect on IBS symptoms (Shi et al., 2008), review of combination therapy (herbal medicine and conventional drugs) in IBS has not provided conclusive evidence of its efficacy, due to small study sample sizes and methodological flaws (Liu et al., 2006). Consequently, the real efficacy of combination therapy involving TCM for IBS is still unclear.

Our meta-analysis evaluated the evidence for the clinical effectiveness of the combination of TCM and Western medicine in the treatment of IBS. To our knowledge, no other similar review was available. In the present study, a total of 72 randomized controlled trials were included for our meta-analysis. We found that TCM combined with Western interventions significantly improved global symptoms of IBS. At the same time, the relapse rate was lower, and no serious adverse events were reported. Additionally, there was no significant difference in therapeutic effects of the integrated approach in the meta-analyses involving the various IBS subtypes (IBS-D, IBS-C, not specify the type of IBS). Similarly, Chinese proprietary herbal medicine plus conventional treatment and compound herbal preparations plus conventional treatment showed similar and statistically significant effects on global improvement compared with Western treatment alone. Evaluating the global improvement of symptoms of all the studies, different subtypes and subgroups, our results demonstrated that the therapeutic effect for relieving IBS symptoms was enhanced by the combination therapy. This may be due to the synergic actions of TCM plus Western medicine. However, further studies investigating the potential mechanisms of pharmacological action in well-designed clinical trials are warranted to elucidate this.

The most striking finding in this review was the assessment of the similar therapeutic effect of Chinese proprietary medicine and compound herbal decoctions. Chinese proprietary medicines, usually formulated as tablets or capsules for convenience or better palatability, can make up for poor adherence of herbal formulae that require boiling the different herbs. Therefore, it is different from decoction which can be modified based on different syndrome, and its application is usually only for patients with specified syndrome. However, in our meta-analysis, 8 Chinese proprietary medicines that supposedly would be suitable for different patients' syndrome types, such as Senling Baizhu granules, Liuwei Anxiao capsules, Shenqi Fuzheng powder, and Gushen Changan capsules, were used in 
12 studies. The result suggests that Chinese proprietary herbal medicines may have similar treatment effect on all IBS subtypes. This would potentially facilitate the use of Chinese proprietary medicine in the management of IBS as not all Chinese proprietary medicine may be readily available, especially outside China. However, due to small study number and sample sizes, further large and rigorous trials are needed to confirm this promising treatment option.

From the public health perspective, in the new National Essential Medicines List issued by the Ministry of Health of China, 203 kinds of Chinese proprietary medicines were selected to meet the most fundamental health needs of the population as essential medicines. To be included into the National Essential Medicines List, a drug should be able to satisfy health care needs and in appropriate dosage forms for administration as well as at a price the public can afford. Globally, there is a paucity of evidence to substantiate the clinical efficacy of TCM for inclusion into the National Essential Medicines List. The results from our meta-analysis showed that the combination of TCM in the essential medicine is beneficial for IBS patients, and can therefore provide an example to justify the inclusion of TCM in the essential medicine list.

However, several limitations of this study should be noted. First, the quality of the included trials was relatively poor. Second, the treatment duration was different across studies, and there was a lack of follow-up in most studies to assess long-term outcomes. Third, the assessment of side effects and recurrent episodes was not detailed and not available from nearly all included studies. Together, these may potentially introduce bias in favor of TCM plus conventional interventions. Furthermore, publication bias should be taken into consideration in this meta-analysis as studies with negative results may not be published or terminated. Finally, we must acknowledge the potential effect of the inability to obtain unpublished literature.

\section{Conclusions}

In summary, the results of this meta-analysis provided the evidence that treating IBS with integrated traditional Chinese and Western medicine showed better effectiveness than conventional Western medicine alone. Our results also showed similar efficacy between Chinese proprietary medicine and herbal decoctions. Chinese proprietary medicine, being low-cost, easy to use, and having good palatability, can make up for poor adherence of Chinese herbal medicine. Therefore, it would be more beneficial and acceptable for IBS patients. The integration of these therapies may provide IBS patients with better treatment outcomes. However, due to the aforementioned limitations, our current data are insufficient to recommend this as first-line treatment. Further multi-center large-scale randomized controlled studies are required to assess the long-term effectiveness and safety of TCM for treatment of IBS.

\section{References}

An, W.Z. and W. Shi. Observation on integrated traditional and Western medicine for treatment of 35 cases of irritable bowel syndrome. J. Yanan Univ. (Med. Sci.) 7: 47-48, 2009. 
Brandt, L.J., W.D. Chey, A.E. Foxx-Orenstein, L.R. Schiller, P.S. Schoenfeld, B.M. Spiegel, N.J. Talley and E.M. Quigley. An evidence-based position statement on the management of irritable bowel syndrome. Am. J. Gastroenterol. 104: S1-S35, 2009.

Cao, X.M. and Y. Ren. Observation on therapeutic effects of integrated traditional and Western medicine for treatment of irritable bowel syndrome. China J. Modern Med. 21: 316-317, 2010.

Chang, J.Y. and N.J. Talley. Current and emerging therapies in irritable bowel syndrome: From pathophysiology to treatment. Trends. Pharmacol. Sci. 31: 326-334, 2010.

Chen, H.S. Clinical analysis of integrated traditional and Western medicine for treatment of diarrheapredominant irritable bowel syndrome. Asia-Pacific Trad. Med. 9: 98-99, 2013.

Chen, M.L. Clinical study on integrated traditional and Western medicine for treatment of 48 cases of irritable bowel syndrome. World Health Dig. 9: 449, 2012.

Chen, Y.J. Clinical observation on Shenling Baizhu granules plus Trimebutine for treatment of irritable bowel syndrome. J. Pract. Trad. Chin. Med. 27: 318-319, 2011.

Chen, Z.S., W.D. Zhang, B.H. Wei and D.B. Li. Irritable bowel syndrome diagnosis and treatment of integrative medicine program (draft). Chin. J. Integr. Trad. West. Med. Dig. 13: 65-67, 2005.

Cheng, Z.S. Sishen Wan modified decoction plus Pinaverium Bromide and Bifidobacterium for treatment of diarrhea-predominant irritable bowel syndrome. Liaoning J. Trad. Chin. Med. 37: 79-80, 2010.

Du, J.S. Observation on therapeutic effects of integrated traditional and Western medicine for treatment of diarrhea-predominant irritable bowel syndrome. J. Emerge. Tradit. Chin. Med. 21: 1521, 2012.

Fan, X.Y. Observation on therapeutic effects of integrated traditional and Western medicine for treatment of 70 cases of constipation-predominant irritable bowel syndrome. J. Community Med. 7: 71-72, 2009.

Grundmann, O. and S.L. Yoon. Complementary and alternative medicines in irritable bowel syndrome: An integrative view. World J. Gastroenterol. 20: 346-362, 2014.

Gu, C., J. Qiao, M. Zhu, J. Du, W. Shang, W. Yin, W. Wang, M. Han and W. Lu. Preliminary evaluation of the interactions of Panax ginseng and Salvia miltiorrhiza Bunge with 5-fluorouracil on pharmacokinetics in rats and pharmacodynamics in human cells. Am. J. Chin. Med. 41: 443-458, 2013.

Hao, Y.W. Clinical observation on combination of traditional Chinese and Western medicine in treatment of irritable bowel syndrome. J. Henan Univer. Sci. Technol. (Med. Sci.) 29: 186187, 2011.

He, J.J. Integrated traditional and Western medicine for treating 68 cases of diarrhea-predominant irritable bowel syndrome. Shandong J. Trad. Chin. Med. 29: 261, 275, 2010.

Hong, C.J. Integrated traditional and Western medicine for treating 25 cases of irritable bowel syndrome with liver stasis and spleen deficiency. Chin. J. Trad. Med. Sci. Technol. 17: 84-85, 2010.

Huang, C.F., H. Jin and Q.Z. Di. Zhenqi Fuzheng powder plus Bifidobacterium for treatment of 64 cases of diarrhea-predominant irritable bowel syndrome. China Pharmaceuticals 20: 75-76, 2011.

Huang, W.D. Observation of therapeutic effects on irritable bowel syndrome treated by Shugan Jieyu capsule plus Pinaverium Bromide. J. Youjiang Med. Univ. Natl. 834-835, 2011.

Ji, F.T. Combination of traditional Chinese and Western medicine for treatment of 28 cases of diarrhea-predominant irritable bowel syndrome. J. Shanxi Coll. Trad. Chin. Med. 33: 16-17, 2010.

Jiang, K.Y. Clinical study of therapeutic effects on constipation-predominant irritable bowel syndrome treated by Runchang Shu recipe combined with Western medicine. Chin. J. Misdiagnostics 9: 6851-6852, 2009. 
Leng, Q.N. Clinical observation of trimebutine maleate in the treating irritable bowel syndrome. China Healthcare Frontiers 4: 32, 40, 2009.

Li, J.W. and Z.C. Wang. 60 Cases of type of diarrhea of irritable bowel syndrome treated in the disperse the depressed liver-energy and invigorate the spleen decoction united Maleic Acid trimebutine. J. Henan Univer. Chin. Med. 24: 69-70, 2009.

$\mathrm{Li}$, L. Treatment of 60 cases of irritable bowel syndrome with integrated traditional and Western medicine. Zhejiang J. Trad. Chin. Med. 47: 520, 2012a.

Li, Q., G.Y. Yang and J.P. Liu. Syndrome differentiation in Chinese herbal medicine for irritable bowel syndrome: A literature review of randomized trials. Evid. Based Compl. Alternat. Med. 2013: 232147, 2013.

Li, Q., Y. Yu and M. Yang. Observation on therapeutic effects of traditional Chinese medicine combined with Mosapride for treatment of constipation-predominant irritable bowel syndrome. Pract. Clin. J. Integr. Trad. Chin. West. Med. 11: 20-21, 2011.

$\mathrm{Li}$, R.P. Clinical observation on therapeutic effects of integrated traditional and Western medicine for treatment of irritable bowel syndrome. Chin. J. Clin. Ration. Drug Use 3: 52-53, 2010.

$\mathrm{Li}, \mathrm{X} . \mathrm{W}$. Clinical observation on comprehensive treatment of 58 cases of irritable bowel syndrome. Hubei J. Trad. Chin. Med. 34: 45, 2012.

Li, Z.M. Clinical observation on integrated traditional and Western medicine for treatment of diarrhea-predominant irritable bowel syndrome. Nei Mongol J. Trad. Chin. Med. 11: 78-79, $2012 b$.

Liao, C.T. Clinical observation on integrated traditional and Western medicine for treatment of constipation-predominant irritable bowel syndrome. China Modern Med. 17: 78, 2010.

Liu, H., Y. Li and F. Liu. Combination of Chinese and Western medicine for treatment of 30 cases of diarrhea-predominant irritable bowel syndrome. J. Pract. Trad. Chin. Inter. Med. 23: 60-61, 2009a.

Liu, J., L.P. Shen and L.T. Weng. Integrated traditional and Western medicine for treatment of 60 cases of constipation-predominant irritable bowel syndrome. Modern J. Integr. Trad. Chin. West. Med. 18: 1019-1020, $2009 \mathrm{~b}$.

Liu, J.P., M. Yang, Y.X. Liu, M. Wei and S. Grimsgaard. Herbal medicines for treatment of irritable bowel syndrome. Cochrane Database Syst. Rev. 1: CD004116, 2006.

Liu, J.S. and X.H. Hou. A review of the irritable bowel syndrome investigation on epidemiology, pathogenesis and pathophysiology in China. J. Gastroenterol. Hepatol. 26: 88-93, 2011.

Liu, S.H., T.H. Lu, C.C. Su, I.S. Lay, H.Y. Lin, K.M. Fang, T.J. Ho, K.L. Chen, Y.C. Su, W.C. Chiang and Y.W. Chen. Lotus leaf (Nelumbo nucifera) and its active constituents prevent inflammatory responses in macrophages via JNK/NF-kappaB signaling pathway. Am. J. Chin. Med. 42: 869-889, 2014a.

Liu, W. and Z. Xu. Clinical observation on Mazi Renwan plus pinaverium bromide for treatment of 50 cases of constipation-predominant irritable bowel syndrome. Guide of China Med. 9: 115116, 2011.

Liu, W.Y., G.Z. Weng and C.Y. Chen. Integrated traditional and Western medicine for treatment of 40 cases of mixed type irritable bowel syndrome. J. Fujian Univer. Trad. Chin. Med. 21: 6061, 2011.

Liu, X.B. and S. Yang. Integrated traditional and Western medicine for treatment of irritable bowel syndrome. World Health Digest 6: 32-33, 2009.

Liu, Y., Y. Huang, C. Zhao, X. Qin, Q. Zhu, S. Chen and J. Qu. Salvia miltiorrhiza injection on pulmonary heart disease: A systematic review and meta-analysis. Am. J. Chin. Med. 42: 13151331, 2014b.

Longstreth, G.F., W.G. Thompson, W.D. Chey, L.A. Houghton, F. Mearin and R.C. Spiller. Functional bowel disorders. Gastroenterology 130: 1480-1491, 2006. 
Lu, L. and J. Yan. Clinical efficacy of Siliankang combined with compound Glutamin capsule in the treatment of diarrhea-predominant irritable bowel syndrome. Modern Trad. Chin. Med. 32: 8$10,2012$.

Lv, R.Y. Clinical observation on integrated traditional and Western medicine for treatment of irritable bowel syndrome. Shiyong Zhenliao 10: 46, 2012.

Miao, Y.Q. Observation on therapeutic effects of integrated traditional and Western medicine for treatment of 38 cases of diarrhea-predominant irritable bowel syndrome. Hunan J. Trad. Chin. Med. 29: 45-46, 2013.

Nellesen, D.,K. Yee, A. Chawla, B.E. Lewis and R.T. Carson. A systematic review of the economic and humanistic burden of illness in irritable bowel syndrome and chronic constipation. $J$. Manag. Care Pharm. 19: 755-764, 2013.

Ou, S.F. Treatment of 40 cases of irritable bowel syndrome with integrated traditional and Western medicine. Hunan J. Trad. Chin. Med. 25: 67-68, 2009.

Pan, G.Z., S.C. Lu, M.Y. Ke, S.M. Han, H.P. Guo and X.C. Fang. An epidemiologic study of irritable bowel syndrome in Beijing: A stratified randomized study by clustering sampling. Chin. J. Epidemiol. 21: 26-29, 2000.

Pang, G.X., H.B. Xu and X.M. Shi. Observation on therapeutic effects of integrated traditional and Western medicine for treatment of 60 cases of diarrhea-predominant irritable bowel syndrome. Hebei J. Trad. Chin. Med. 33: 1027, 1052, 2011.

Quigley, E.M., P. Bytzer, R. Jones and F. Mearin. Irritable bowel syndrome: The burden and unmet needs in Europe. Digest. Liver Dis. 38: 717-723, 2006.

Rahimi, R. and M. Abdollahi. Herbal medicines for the management of irritable bowel syndrome: A comprehensive review. World J. Gastroenterol. 18: 589-600, 2012.

Ren, M.J. and X.Y. Jiang. Observation of therapeutic effects on diarrhea-predominant irritable bowel syndrome treated by compound glutamine enter soluble capsules plus trimebutine. Modern J. Integr. Trad. West. Med. 20: 833-834, 2011.

Ringel, Y., R.E. Williams, L. Kalilani and S.F. Cook. Prevalence, characteristics, and impact of bloating symptoms in patients with irritable bowel syndrome. Clin. Gastroenterol. Hepatol. 7: 68-72, 2009.

Shen, J.C., G.H. Guo and S.Y. Ye. Observation of therapeutic effects on irritable bowel syndrome treated by Zhizhu Kuanzhong capsule. Zhejiang J. Trad. Chin. Med. 47: 99, 2012.

Shergis, J.L., A.L. Zhang, W. Zhou and C.C. Xue. Quality and risk of bias in Panax ginseng randomized controlled trials: A review. Am. J. Chin. Med. 41: 231-252, 2013.

Shi, J., Y. Tong, J.G. Shen and H.X. Li. Effectiveness and safety of herbal medicines in the treatment of irritable bowel syndrome: A systematic review. World J. Gastroenterol. 14: 454-462, 2008.

Song, X.R. Xiaojian Zhong decoction plus Pinaverium Bromide for treating 33 cases of irritable bowel syndrome with spleen deficiency. Yunnan J. Trad. Chin. Med. 34: 37-38, 2013.

$\mathrm{Su}, \mathrm{M} .42$ cases of diarrhea-predominant irritable bowel syndrome treated in the integrative medicine. Clin. J. Chin. Med. 3: 99, 101, 2011.

Sun, C.X. Diagnostic Basis of Clinical Disease and Criteria for Recovery and Improvement. People's Military Medical Press, Beijing, 2002, pp. 77-78.

Sun, S.H. Integrated traditional and Western medicine for treatment of 30 cases of diarrhea-predominant irritable bowel syndrome. China's Naturopathy 21: 51, 2013.

Sun, Y.L. and D.X. Zhao. Treatment of irritable bowel syndrome with modified Tongxie YaoFang: A 83 cases study. Int. J. Trad. Chin. Med. 31: 429-430, 2009.

Tang, Z.P. Traditional Chinese medicine clinical experience of the treatment for irritable bowel syndrome. Chin. J. Integr. Med. 15: 93-94, 2009.

Tong, X.F. and Y.C. Cai. Clinical observation on integrated traditional and Western medicine for treating 102 cases of diarrhea-predominant irritable bowel syndrome. Hebei J. Trad. Chin. Med. 34: 874-875, 2012. 
Wang, D.B. Treatment of IBS with integrated traditional Chinese and Western medicine. Med. J. Chin. People's Health 21: 73-74, 2009a.

Wang, H.L. Clinical observation on integrated traditional and Western medicine for treatment of 76 cases of irritable bowel syndrome. Hebei J. Trad. Chin. Med. 33: 1514-1515, 2011.

Wang, S.F. and Z.X. Ji. Integrated traditional and Western medicine for treatment of 23 cases of irritable bowel syndrome. Chin. J. Clinicians 37: 40-41, 2009.

Wang, S.H. Observation of therapeutic effects on integrated traditional and Western medicine for treatment of irritable bowel syndrome. China Foreign Med. Treat. 18: 80, $2009 \mathrm{~b}$.

Wang, X. and J.M. Zhu. Clinical experience on integrated traditional Chinese and Western medicine in treatment of 58 patients with constipation type irritable bowel syndrome. J. Modern Med. Health 25: 2260-2261, 2009.

Wang, Z.J., H.X. Li, J.H. Wang and F. Zhang. Effect of Shugan Jianpi Granule on gut mucosal serotonin-positive cells in patients with irritable bowel syndrome of stagnated Gan-qi attacking Pi syndrome type. Chin. J. Integr. Med. 14: 185-189, 2008.

Wei, Y., S.L. Huang, W.S. Zhang, S.N. Deng, F. Feng and L.X. Hu. Clinical observation on integrated traditional and Western medicine for treatment of irritable bowel syndrome. Liaoning J. Trad. Chin. Med. 37: 2417-2418, 2010.

Wen, X.D., C.Z. Wang, C. Yu, Z. Zhang, T. Calway, Y. Wang, P. Li and C.S. Yuan. Salvia miltiorrhiza (dan shen) significantly ameliorates colon inflammation in dextran sulfate sodium induced colitis. Am. J. Chin. Med. 41: 1097-1108, 2013.

Weng, L.T., L.P. Shen and J.P. Liu. Clinical observation on integrated traditional and Western medicine for treatment of 32 cases of irritable bowel syndrome. Hebei J. Trad. Chin. Med. 31: 251-252, 2009.

Wu, G.Q. Clinical observation on integrated traditional and Western medicine for treatment of 35 cases of diarrhea-predominant irritable bowel syndrome. Guiding J. Trad. Chin. Med. Pharm. 15: 29-30, 2009.

Wu, S.C. and Z.J. Zheng. Observation on curative effect on 30 cases of irritable bowel syndrome treated by combination of TCM with Western medicine. Clin. J. Chin. Med. 2: 36, 42, 2010.

$\mathrm{Wu}$, X.H., Z.L. Wang and J.Y. Wang. Treatment of 67 cases of irritable bowel syndrome with integrated traditional and Western medicine. Chin. Foreign Med. Res. 8: 29-30, 2010.

Xie, D.Y. and X. Chen. Combination of traditional Chinese and Western medicine for treatment of 52 cases of diarrhea-predominant irritable bowel syndrome. Clin. J. Trad. Chin. Med. 21: 26-27, 2009.

Xiong, B. Treatment of 56 cases of irritable bowel syndrome with integrated traditional and Western medicine. Chin. Med. Modern Distance Edu. Chin. 11: 63, 2013.

Xiong, L.S., M.H. Chen, H.X. Chen, A.G. Xu, W.A. Wang and P.J. Hu. A population-based epidemiologic study of irritable bowel syndrome in Guangdong Province. Natl. Med. J. Chin. 84: 278-281, 2004.

Xiong, W.S. Study on integrated traditional and Western medicine for treatment of irritable bowel syndrome. World Health Digest 8: 287-288, 2011.

$\mathrm{Xu}$, W.Q. and R.M. Wang. Observation on therapeutic effects of integrated traditional and Western medicine for treatment of 21 cases of constipation-predominant irritable bowel syndrome. Forum on Trad. Chin. Med. 26: 32-33, 2011.

Yan, T.X. Clinical study on integrated traditional and Western medicine for treatment of irritable bowel syndrome. Chin. J. Clin. Ration. Drug Use 2: 59-60, 2009.

Yang, J. Observation on therapeutic effects of integrated traditional and Western medicine for treatment of irritable bowel syndrome. Seek Medicial and Ask the Medicine 10: 153-154, 2012.

Ye, F., Y.X. Zhang, J.L. Yang and D.X. Li. Clinical observation on integrated traditional and Western medicine for treatment of 72 cases of irritable bowel syndrome. Hebei J. Trad. Chin. Med. 34: 1830-1831, 2012. 
Yi, S., Y.Q. Zhang and H. Lu. Yijining Tang for treatment of 42 cases of irritable bowel syndrome. $J$. Pract. Trad. Chin. Int. Med. 23: 55-56, 2009.

Yin, Y.L. and M.G. Dong. Clinical observation of irritable bowel syndrome treated with integrated traditional Chinese and Western medicine. Chin. J. Integr. Trad. West. Med. Dig. 18: 37-39, 2010.

Yoon, S.L., O. Grundmann, L. Koepp and L. Farrell. Management of irritable bowel syndrome (IBS) in adults: Conventional and complementary/alternative approaches. Altern. Med. Rev. 16: 134$151,2011$.

$\mathrm{Yu}$, J.H. and H.Lao. Observation of therapeutic effects on 36 cases of diarrhea-predominant irritable bowel syndrome treated by berberine plus compound glutamine enter soluble capsules. $J$. Guangdong Med. Coll. 30: 427-428, 2012.

$\mathrm{Yu}$, W.G., G. Chen and Q.J. Liu. Integrated traditional and Western medicine for treatment of 41 cases of diarrhea-predominant irritable bowel syndrome. Chin. Manipul. Rehabil. Med. 7: 174, 2011.

Zeng, B.T. Treatment of 66 cases of irritable bowel syndrome with integrated traditional and Western medicine. Hunan J. Trad. Chin. Med. 25: 65-66, 2009.

Zeng, Y.M. Clinical observation on integrated traditional and Western medicine for treatment of 45 cases of irritable bowel syndrome. Chin. J. Integr. Trad. West. Med. Inten. Crit. Care 18: 319, 2011.

Zeng, Z. and H.H. Yan. Effect observation of Tongxie Yaofang and Pinaverium Bromide tablets in treatment of irritable bowel syndrome. China Med. Pharm. 2: 27-28, 2012.

Zhang, B.H., R. Gao, Z.H. Li, B.S. Li, F.Y. Wang and X.D. Tang. Treatment of irritable bowel syndrome by Chinese medicine and pharmacy: An analysis of data mining on experiences of experts. Chin. J. Integr. Trad. West. Med. 33: 757-760, 2013.

Zhang, L.M. Clinical study of therapeutic effects on diarrhea-predominant irritable bowel syndrome treated by integrated traditional and Western medicine. Guide of China Medicine 10: 279-280, 2012.

Zhang, Y.F., Z. Zhang and X.R. Zheng. Clinical observation on integrated traditional and Western medicine for treatment of diarrhea-predominant irritable bowel syndrome. Chin. Pract. J. Rural Doctor 16: 34-35, 2009.

Zhao, H.L., C.Z. Sun, W.P. Jiang, Z.K. Dai, W.X. Shi, K.K. Yang, X.J. Mu, X.X. Zhang and Y. Sui. Eight-year survival of AIDS patients treated with Chinese herbal medicine. Am. J. Chin. Med. 42: 261-274, 2014.

Zhao, M.L. Clinical observation on integrated traditional and Western medicine for treatment of irritable bowel syndrome. Hebei J. Trad. Chin. Med. 31: 1342-1343, 2009.

Zhen, Q.F. Integrated traditional and Western medicine for treatment of 30 cases of diarrhea Predominant irritable bowel syndrome. Hebei J. Trad. Chin. Med. 31: 248-249, 2009.

Zheng, F.S. Integrated traditional and Western medicine for treatment of 48 cases of irritable bowel syndrome. Yiayao Qianyan 6: 305-306, 2013a.

Zheng, F.S. Observation on therapeutic effects of combination of TCM with Western medicine for treatment of 180 cases of irritable bowel syndrome. World Health Digest 10: 177-178, 2013b.

Zheng, X.Y. Clinical Research Guidelines for New Chinese Herbal Drug. China Medicial Science Press, Beijing, China, 2002, pp. 140-143.

Zhou, H.F., A.J. Xu and Y.X. Liao. Clinical efficacy of treating irritable bowel syndrome with combination of traditional Chinese and Western medicine. Chin. Arch. Trad. Chin. Med. 30: 652-654, 2012.

Zhu, K.D. Clinical observation on compound glutamine enter soluble capsules plus Bifidobacterium for treating diarrhea-predominant irritable bowel syndrome. Chin. J. Gener. Pract. 10: 238, 263, 2012. 
Copyright of American Journal of Chinese Medicine is the property of World Scientific Publishing Company and its content may not be copied or emailed to multiple sites or posted to a listserv without the copyright holder's express written permission. However, users may print, download, or email articles for individual use. 